\title{
Medial Image of the Week: Purpura Fulminans
}

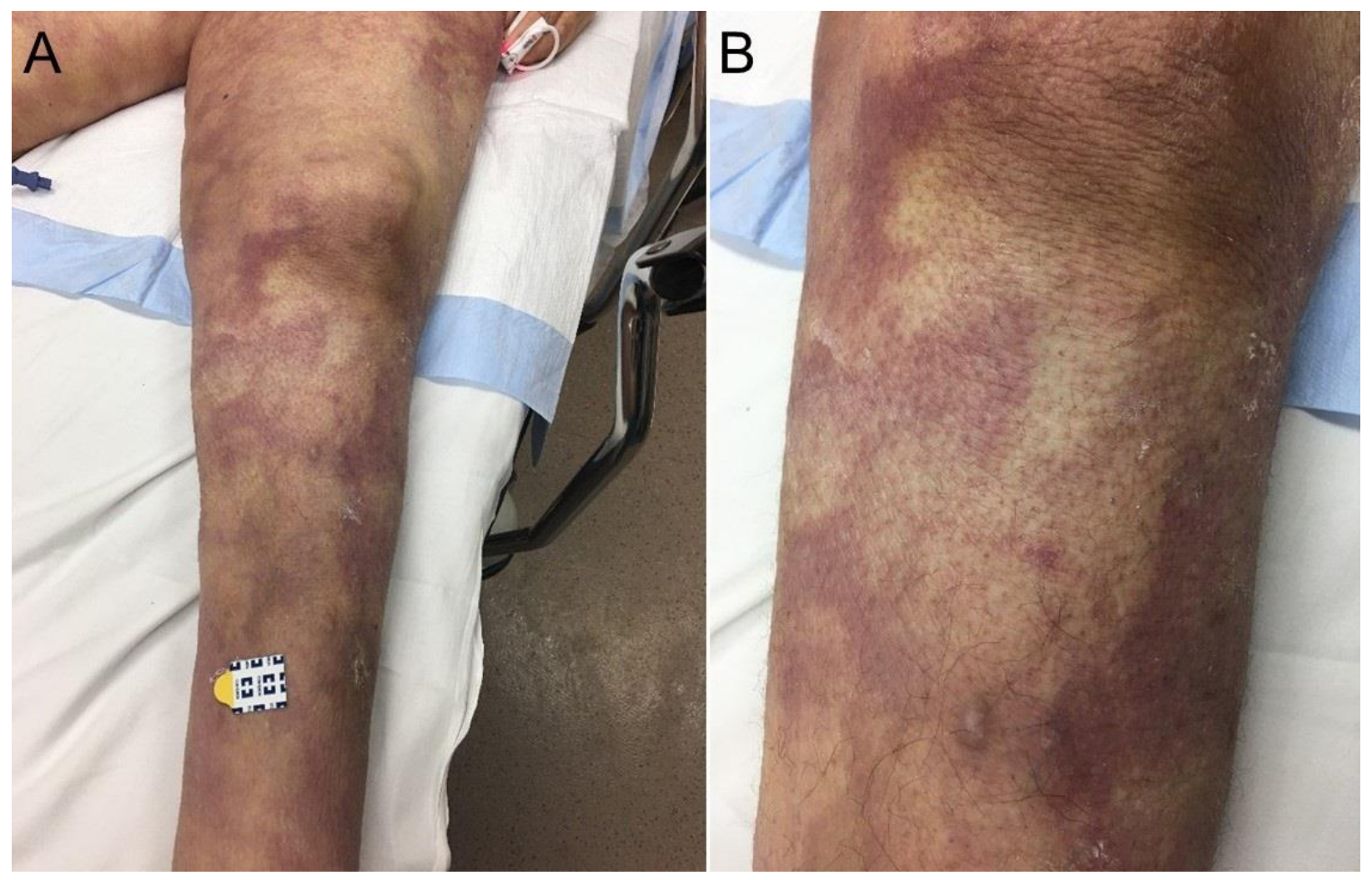

Figure 1. A: Purpura fulminans. B: Close up view of the left lower extremity.

A 54-year-old man with coronary artery disease, fibromyalgia and chronic sacral ulcers was brought to the emergency department due to acute changes in mentation and hypotension. He suffered a cardiac arrest shortly after arrival to the emergency department during emergent airway management. After successful resuscitation, he was admitted to the medical intensive care unit and treated for septic shock with fluid resuscitation, vasopressors and broad spectrum antibiotics. Laboratory results were significant for disseminated intravascular coagulopathy (DIC)- thrombocytopenia, decreased fibrinogen and elevated PT, PTT and D-dimer levels. Profound metabolic acidosis and lactate elevation was also seen. Blood Cultures later revealed a multi-drug resistant $E$. coli bacteremia. Images of the lower extremities (Figure 1) were obtained at initial assessment and are consistent with purpura fulminans. He did not survive the stay.

Purpura fulminans, also referred to as skin mottling, is an evolving skin condition which is characterized by an acutely worsening reticular pattern of ecchymosis, tissue thrombosis and eventual hemorrhagic skin necrosis. Traditionally associated with either an inherited and/or acquired protein C deficiency, it is more commonly seen in DIC. It is generally considered a poor prognostic indicator when associated with DIC. In our 
patient, the DIC was secondary to septic shock. When encountered in this clinical scenario it should be considered an acute life threatening emergency.

Emilio Perez Power MD ${ }^{1}$, Norman Beatty $\mathrm{MD}^{1}$ and Bhupinder Natt MD ${ }^{2}$

${ }^{1}$ Department of Internal Medicine and ${ }^{2}$ Division of Pulmonary, Allergy, Critical Care and Sleep

Banner-University Medical Center South Campus

University of Arizona

Tucson, AZ USA 